\section{A computer program that produces line graphs}

\author{
NEAL L. KANE \\ Iowa Mental Health Authority \\ University of Iowa, Oakdale, Iowa 52319
}

Description. The program described here can plot line graphs suitable for publication in journal articles. Several computer-generated graphs were used in an article by Kane and Knutson (1976). An example of a line graph produced by the program is shown in Figure 1. As part of each graph, the program prints a legend with labels for the solid and/or dashed lines being plotted. The program can also print a caption underneath the graph. The use of this feature is optional; graphs with or without captions can be produced. The user has flexibility in plotting the $\mathrm{X}$ axis and $\mathrm{Y}$ axis for each graph, since the user specifies such information as the number of divisions (tick marks) on each axis, the distance between the divisions, the labels for the divisions, and the labels for the axes.

Input. The user first provides data that are not specific to any single graph being plotted. These data include: identifying information about the user, such as the user's name and identification number; the plotting symbols to be used in representing the data points on the graphs (e.g., triangle, square, or octagon); and the

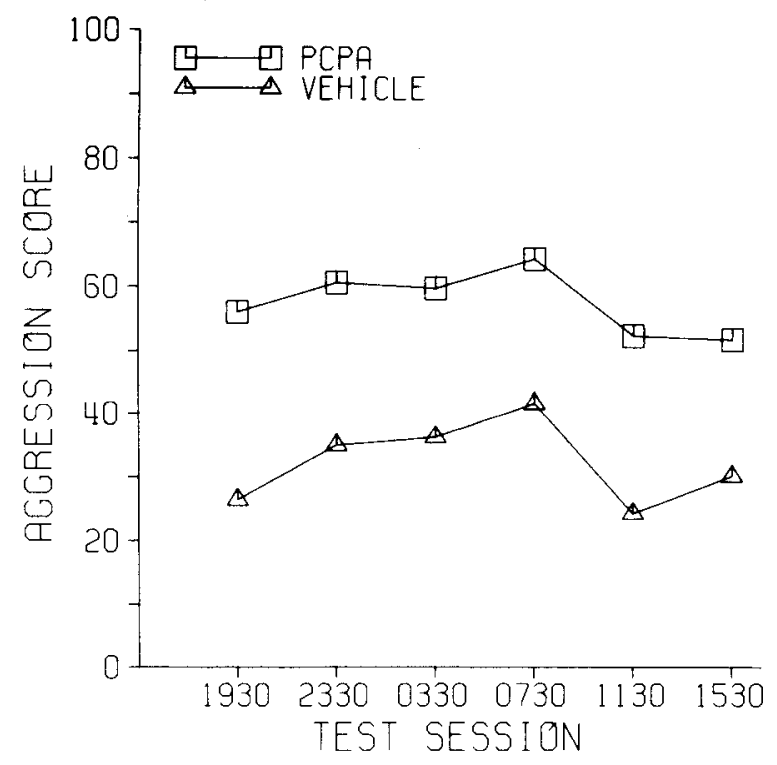

Figure 1. Example of a graph produced by the computer program. number of graphs being plotted. Next, the data for the first graph are entered. Values are provided for such parameters as the number of solid lines, the number of dashed lines, and the number of points to be plotted for each line. The user then enters information for the first graph, which is used to plot the graph legend, the $\mathrm{X}$ axis, the $Y$ axis, the solid and/or dashed lines, and the graph caption, if a caption is requested. This information is repeated for each graph to be plotted.

Output. The program prints out the raw data entered by the user. This printout is designed to assist the user to find and correct any mistakes made in entering the data. At the University of Iowa, the graphs can be produced on either microfilm or white paper. The microfilm graphs can be used as slides. Black-and-white prints can be made from the microfilm. Such prints were used in the Kane and Knutson (1976) article.

Language and Computer. The program is written in FORTRAN IV, Level G, for the IBM 360/65 and makes use of various plotting subroutines supplied by California Computer Products, Inc. (CalComp). An on-line CalComp 855 microfilm plotter and an off-line IBM 1627 drum plotter are available for plotting at the Weeg Computer Center at the University of Iowa.

Limitations. The plotting program uses primarily lower level plotting subroutines that draw lines, character strings, and plotting symbols. It is likely that the plotting subroutines can be used at most computer installations that have plotting facilities. The only subroutine that is not at this lower level is subroutine DASHLN, which plots dashed lines. A local plotting reference manual should be consulted to determine if each plotting subroutine exists in the same form at the user's computer installation. The documentation for the program includes a description of the CalComp plotting subroutines used.

Availability. A listing of the plotting program, instructions for entering data, a listing of the program variables for which the user provides values, and a description of the CalComp plotting subroutines used in the program are available free of charge from the author at the Iowa Mental Health Authority, the University of Iowa, Oakdale Campus, Oakdale, Iowa 52319.

\section{REFERENCE}

Kane, N. L., \& KNUtson, J. F. Influence of colony lighting conditions on home-cage spontaneous aggression. Journal of Comparative and Physiological Psychology, 1976, 90, 889-897.

(Accepted for publication June 14, 1979.) 